\title{
OPEN Characterization of 3D-printed PLA parts with different raster orientations and printing speeds
}

\begin{abstract}
Mohammad Reza Khosravani ${ }^{1 凶}$, Filippo Berto ${ }^{2}$, Majid R. Ayatollahi ${ }^{3}$ \& Tamara Reinicke $^{1}$
Fabrication based on additive manufacturing (AM) process from a three-dimensional (3D) model has received significant attention in the past few years. Although 3D printing was introduced for production of prototypes, it has been currently used for fabrication of end-use products. Therefore, the mechanical behavior and strength of additively manufactured parts has become of significant importance. 3D printing has been affected by different parameters during preparation, printing, and post-printing processes, which have influence on quality and behavior of the additively manufactured components. This paper discusses the effects of two printing parameters on the mechanical behavior of additively manufactured components. In detail, polylactic acid material was used to print test coupons based on fused deposition modeling process. The specimens with five different raster orientations were printed with different printing speeds. Later, a series of tensile tests was performed under static loading conditions. Based on the results, strength and stiffness of the examined specimens have been determined. Moreover, dependency of the strength and elastic modulus of 3D-printed parts on the raster orientation has been documented. In the current study, fractured specimens were visually investigated by a free-angle observation system. The experimental findings can be used for the development of computational models and next design of structural components.
\end{abstract}

Additive manufacturing (AM) also known as three-dimensional (3D) printing covers a set of techniques which utilized layer-by-layer concept to fabricate components. 3D printing technology is vastly used in different applications, such as aerospace, automotive, electronics, construction, and medicine, and healthcare monitoring ${ }^{1-6}$. Due to favorable properties of 3D printing technology, it has attracted great interest in various technological sectors.

Based on the documented applications 3D printing, reduction in time and cost by eliminating expensive manufacturing equipment, and possibilities on easy fabrication of geometrically complex components have been considered as important advantages of 3D printing technology. Moreover, in this rapid prototyping process, large reduction of waste material can be achieved, because manufacturing tools are not required, and filed prints can be recycled in an easy and fast process. Production of light structural components with desired weight and utilizing multiple materials at the same time are advantages of $3 \mathrm{D}$ printing compared to traditional manufacturing processes.

As applications of AM have been significantly increased, different engineering aspects have been studied in this field ${ }^{7-10}$. Although $3 \mathrm{D}$ printing was introduced for production of prototypes, it is being used to fabricate final products. In this case, 3D-printed parts and structural elements might be subjected to special loading or environmental conditions. Therefore, it is necessary to investigate mechanical behavior of these components. In this context, several studies have been conducted to determine response of 3D-printed materials to different loading conditions, such as bending, tensile, and torsion ${ }^{11-13}$. For instance, in Ref. ${ }^{14}$ dynamic behavior of 3D-printed reinforced composites was investigated. In detail, fused filament fabrication process was used to produce short carbon-fiber-reinforced composite specimens which are subjected to dynamic loading by a Hopkinson bar. The obtained results indicated that infill density has important role in deformation and strength, but low density and high density infills are cost effective compared to solid components.

Considering applications of 3D-printed components, their structural integrity become an important issue. Hence, fracture behavior of these parts are investigated in several studies ${ }^{15-20}$. In Ref. ${ }^{21}$ fracture behaviors of $3 \mathrm{D}$ printed components have been reviewed. In previous studies, fracture mechanics approach was used for

${ }^{1}$ Chair of Product Development, University of Siegen, Paul-Bonatz-Str. 9-11, 57068 Siegen, Germany. ${ }^{2}$ Department of Mechanical and Industrial Engineering, Norwegian University of Science and Technology (NTNU), 7491 Trondheim, Norway. ${ }^{3}$ Fatigue and Fracture Research Laboratory, Center of Excellence in Experimental Solid Mechanics and Dynamics, Iran University of Science and Technology, Narmak 16846, Tehran, Iran. ${ }^{\square}$ email: mohammadreza.khosravani@uni-siegen.de 


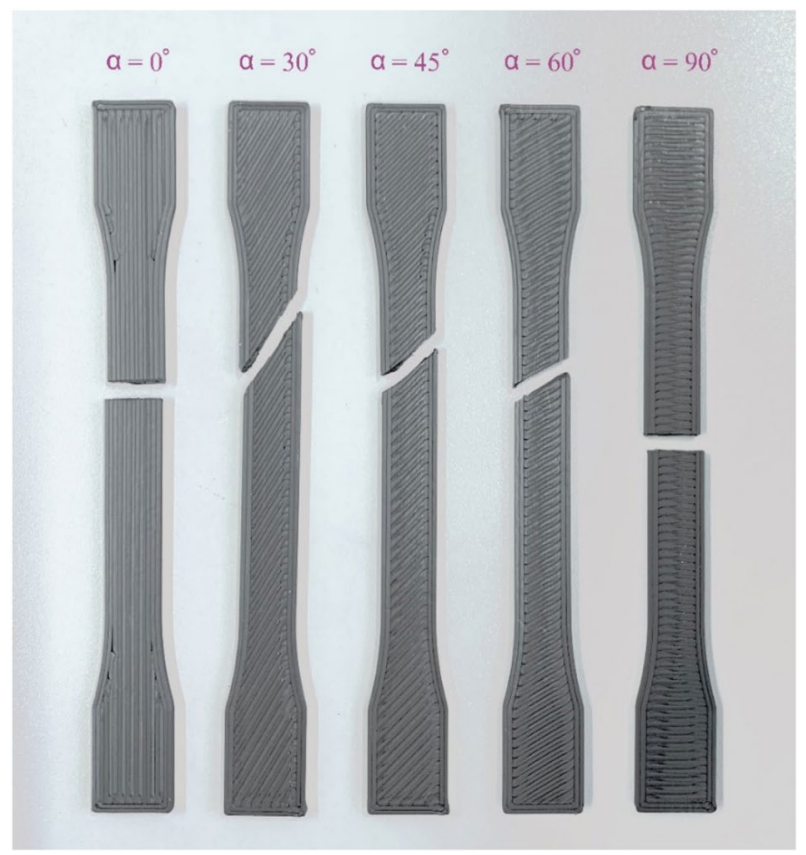

Figure 1. Fractured specimens with different raster directions, printed at $20 \mathrm{~mm} / \mathrm{s}$.

characterization of additively manufactured components ${ }^{22-26}$. For instance, in Ref. ${ }^{27}$ effects of filament-scale geometric features have been investigated. More in deep, 3D-printed compact tension test coupons were examined with different fiber directions. The obtained results confirmed that higher strength is achievable with loading in the filament direction. Moreover, fractography indicated that interfacial weakness leads to anisotropy and change mechanical performance of the component.

Parallel to the experimental tests, numerical models and different artificial intelligence approaches have been used to investigate performance characteristics of 3D-printed components. For instance, in Ref. ${ }^{28}$ back propagation neural network was used to predict printable bridge length in fused deposition modeling (FDM) process. Recently, in Ref. ${ }^{29}$ we have reviewed applications of machine learning in prediction of mechanical behavior of 3D-printed parts. In order to enhance strength and improve mechanical performance of 3D-printed components, different attempts have been made ${ }^{30-33}$. For example, in Ref. ${ }^{31}$ annealing was performed to increase strength of 3D-printed parts. In this context, polymeric materials with different carbon fiber reinforced polymers were utilized to print dog-bone specimens based on material extrusion technique. According to the experimental practices, it has been concluded that annealing is a suitable post-processing technique to enhance interlayer tensile strength of 3D-printed composites. Since, mechanical behavior of 3D-printed components depends on various parameters, a significant amount of attempts has been made in this field ${ }^{34-37}$. Recently, in Ref. ${ }^{38}$ physicochemical and mechanical behavior of PLA matrix in 3D printed composite was investigated. At the same time, in Ref. ${ }^{39}$ FDM process was used to prepare specimens and effects of printing parameters such as nozzle temperature and layer thickness on flexural properties and impact strength of specimens were investigated.

In the current study, we investigated effects of two printing parameters on strength of 3D-printed parts: (a) raster orientation and (b) printing speed. To this aim, test coupons with various raster directions were printed with different speeds. We used PLA bioepolymer with grade of 4043D. Currently, PLA with different grades are used in 3D printing, and results of tests on a particular grade cannot be used for analysis of other grades, therefore, separate research studies are required to determine exact behavior of each grade. Here, a series of tensile tests were conducted and mechanical behavior and strength of the examined parts were documented. Although influence of printing parameters on the mechanical behavior of 3D-printed components have been discussed in some previous studies, to the best of the authors knowledge, experimental tests and two different theoretical formulas are not used on a particular research work with this grade of material which is investigated in the present study. Since creating a strong and dimensionally stable parts have been a challenging goal of FDM technology, study effect of manufacturing parameters on 3D-printed parts is a necessity.

\section{Results and discussion}

Experimental practices confirmed that there was a sudden failure in the specimens after reaching the maximum stress. We documented rather brittle behavior in the examined specimens. In Fig. 1 a close-up pictures of the fractured specimens illustrated. Based on the performed tests, the mechanical behavior of the test coupons are determined. In Fig. 2 stress-strain curves of the examined specimens are illustrated. According to the results, strength and stiffness depend on the raster direction and printing speed.

In the present study, each sample set consisted of six specimens for a given group of process parameters (raster direction and printing speed), with a total of 60 specimens. All specimens were printed using Ender-3 Pro 3D Printer. Figure 2a shows resulting stress-strain curves of the specimens with different raster directions 


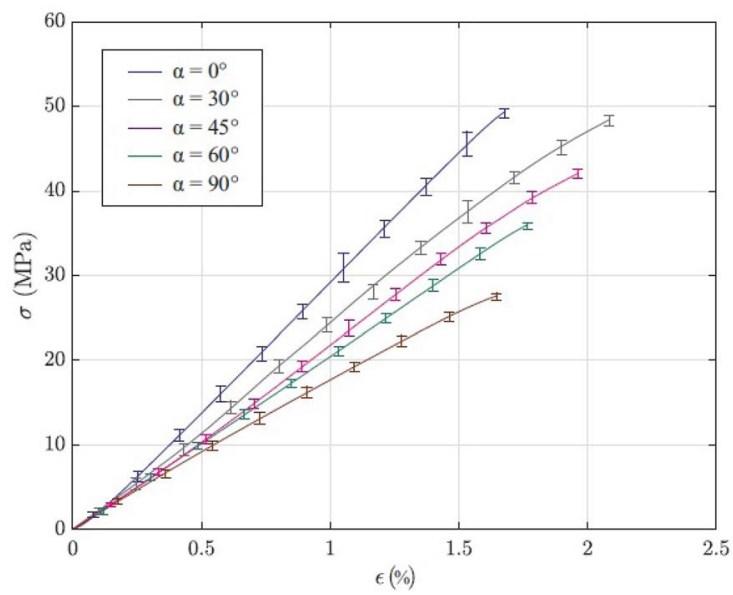

(a)

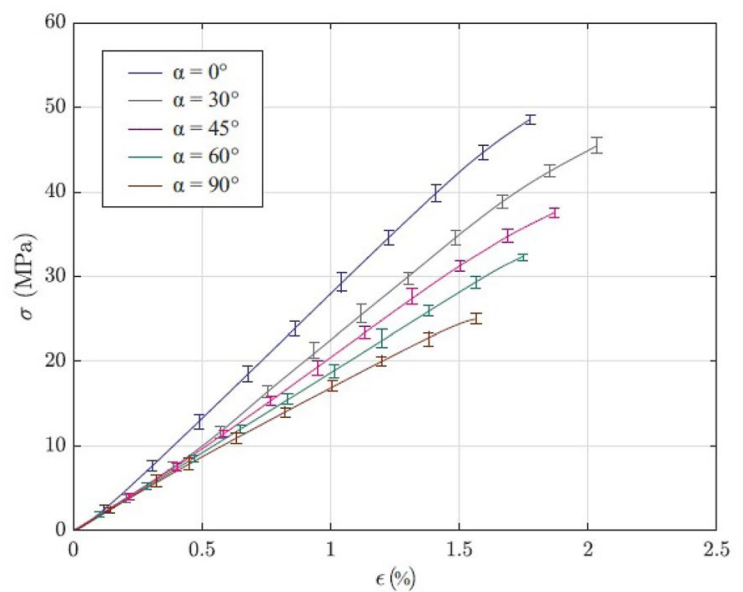

(b)

Figure 2. Stress-strain curves of the tested specimens, printed with different speeds: (a) $20 \mathrm{~mm} / \mathrm{s}$; (b) $80 \mathrm{~mm} / \mathrm{s}$.

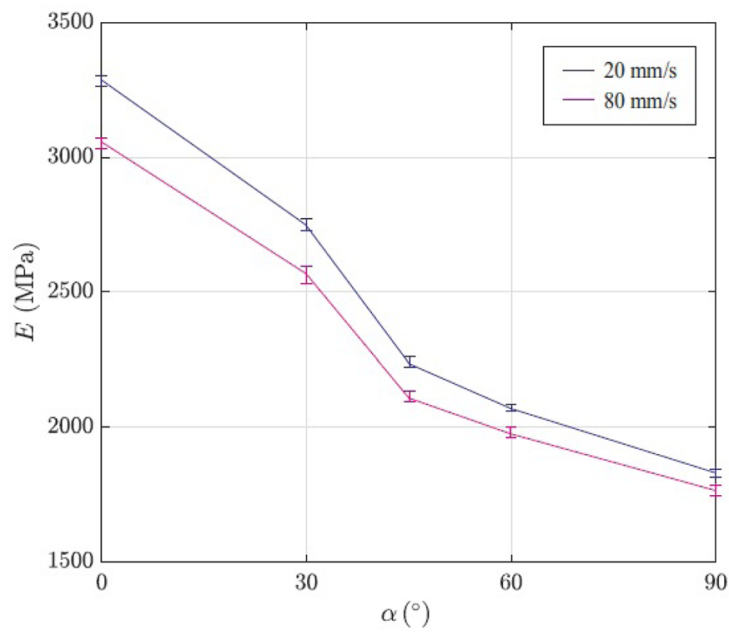

(a)

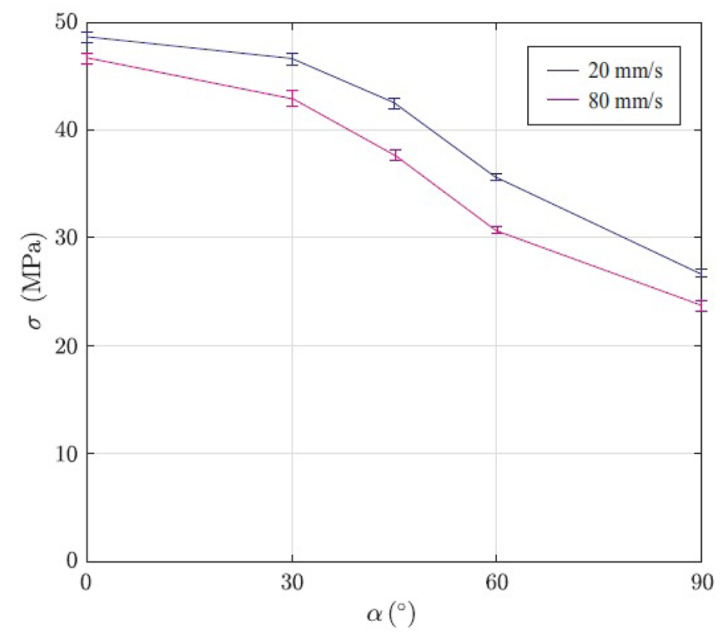

(b)

Figure 3. Dependency of (a) elastic modulus, and (b) tensile strength on the raster direction for the 3D-printed specimens.

which are printed at $20 \mathrm{~mm} / \mathrm{s}$. It can be seen that $0^{\circ}$ and $90^{\circ}$ specimens indicated highest and lowest strengths, respectively. Additionally, Fig. $2 \mathrm{~b}$ shows stress-strain curves of the specimens printed at $80 \mathrm{~mm} / \mathrm{s}$. As can be seen, an increase in the raster direction has led to decrease in strength of the 3D-printed parts. It is noteworthy that printing speed has effect on the elongation of examined specimens, but this influence is small compared to effect of raster direction on the mechanical behavior of 3D-printed components.

According to the experimental findings, we have determined dependency of the elastic modulus and the tensile strength on the raster direction. In Fig. 3a relation between the raster direction and elastic modulus is depicted. As it is shown, an increase in the raster orientation has led to a decrease in elastic modulus of the examined $3 \mathrm{D}$-printed specimens. Hence, $90^{\circ}$ specimens presented lowest elastic modulus. Figure $3 \mathrm{~b}$ shows dependency of tensile strength on raster orientation. The obtained results proved that tensile strength gradually decreased with an increase in the raster orientation.

Since FDM widely used in different applications, various aspects have been investigated in this process ${ }^{40-42}$. For instance, in Ref. ${ }^{42}$ finite element analysis of 3D printing based on FDM process was investigated. In detail, researchers studied impact of several parameters and modeling choices on the simulation findings. Here, based on the results, we concluded that raster direction has a significant effect on the strength of the specimens compared to printing speed. However, at the higher printing speed there is a shorter forming time which has a negative effect on the strength of printed specimens.

Analysis of fracture in the specimens indicated that the interlayer and in-layer fractures were occurred in the specimens after tensile tests. These fractures are defined as follows: 


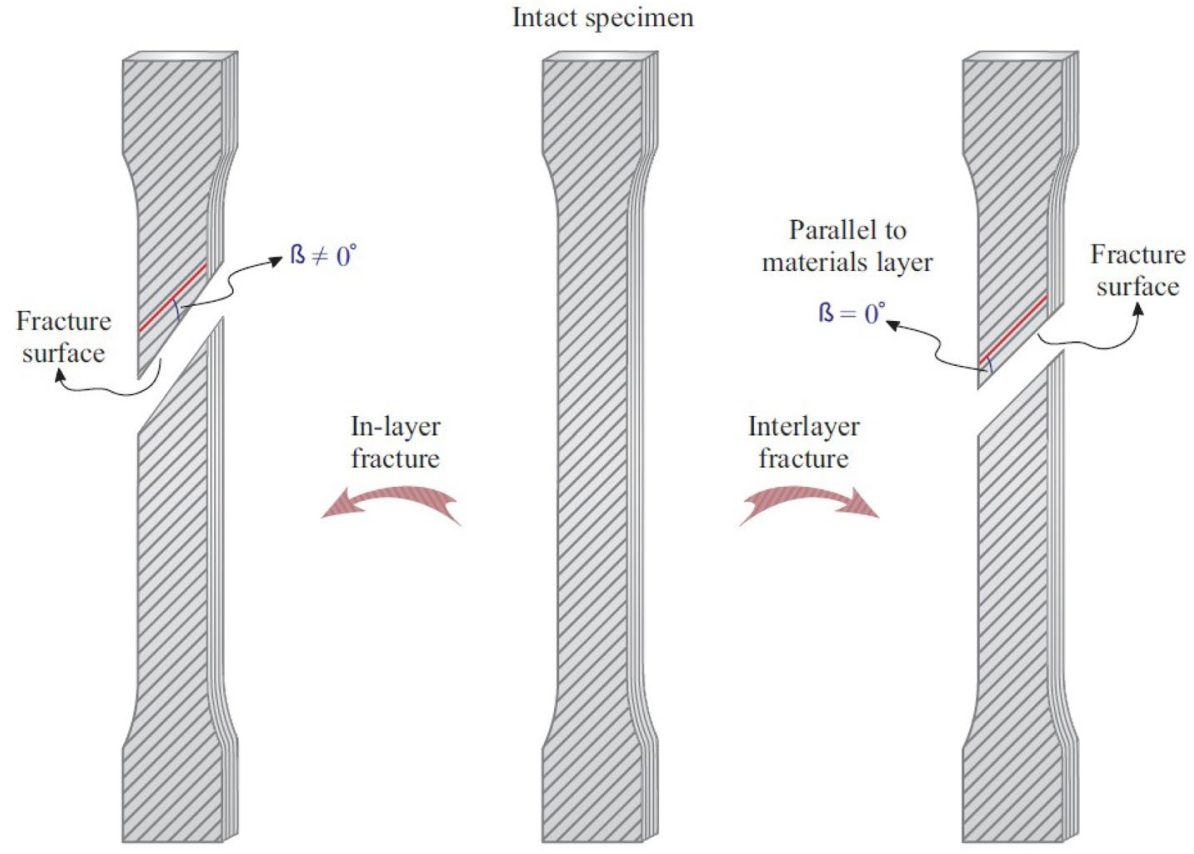

Figure 4. Schematics of interlayer and in-layer fractures.

\begin{tabular}{|l|l|l|l|l|l|}
\hline \multirow{2}{*}{ Printing speed $(\mathbf{m m} / \mathbf{s})$} & \multicolumn{5}{|l|}{ Raster direction } \\
\cline { 2 - 6 } & $\mathbf{0}^{\circ}(\%)$ & $\mathbf{3 0}^{\circ}(\mathbf{\%})$ & $\mathbf{4 5}^{\circ}(\%)$ & $\mathbf{6 0}^{\circ}(\%)$ & $\mathbf{9 0}^{\circ}(\%)$ \\
\hline 20 & 100.00 & 95.83 & 87.51 & 72.91 & 58.33 \\
\hline 80 & 100.00 & 92.47 & 81.72 & 68.81 & 53.76 \\
\hline
\end{tabular}

Table 1. Reduction in the UTS of different 3D-printed specimens.

- Interlayer fracture: in this type of fracture, the angle $(\beta)$ between the materials layer and fracture surface of the materials is equal to zero. In this case, the materials layer would be intact after fracture.

- In-layer fracture: in this fracture, the angle $\beta$ is larger than zero and the materials layer would not be intact after the fracture.

In Fig. 4 interlayer and in-layer fractures are schematically shown. In the current study, both interlayer and in-layer fractures were occurred.

As mentioned earlier, the results showed that the UTS of 3D-printed parts decreased when raster direction increased from $0^{\circ}$ to $90^{\circ}$. Here, we have considered the UTS of the $0^{\circ}$ specimens as a standard (100\%) and determine reduction coefficient of the examined parts with different raster directions as follows:

$$
\text { Reduction coefficinet in UTS }=\frac{\text { UTS }}{\text { UTS in } 0^{\circ} \text { specimen }} .
$$

Considering Eq. (1), reduction in the UTS of different specimens are summarized in Table 1. Experimental results indicated that the reduction in the UTS varies from 53.76 to $100 \%$. In detail, specimen printed at $80 \mathrm{~mm} / \mathrm{s}$ with $90^{\circ}$ raster direction showed smallest reduction coefficient.

Based on the current experimental results, theoretical models are evaluated. In Fig. 5 results of both theoretical models and experimental findings are compared. It can be clearly seen that the both theoretical models Eqs. (11) and (12) predicted the experimental results accurately.

Comparison of the experimental findings and theoretical models confirmed ability of both theoretical models in prediction of UTS of 3D-printed components.

As there are different grades of PLA grades on the market, precise investigations are required to identify mechanical behavior of each grade. In the presented study, we used PLA bioepolymer with grade of 1043D. Based on the previous research by Bermundez et al. ${ }^{43}$ PLA with different grades showed different ultimate tensile strengths. Different types of manufacturing defects can significantly change the mechanical behavior of the components. As different defects and anomalies might be occurred during 3D printing process (e.g., overlap, missing extrudates, and offset), the specimens are visually investigated prior to the tensile test and we have not found any type of defect. After tensile tests, a series of fractographic examination was performed for analysis of 


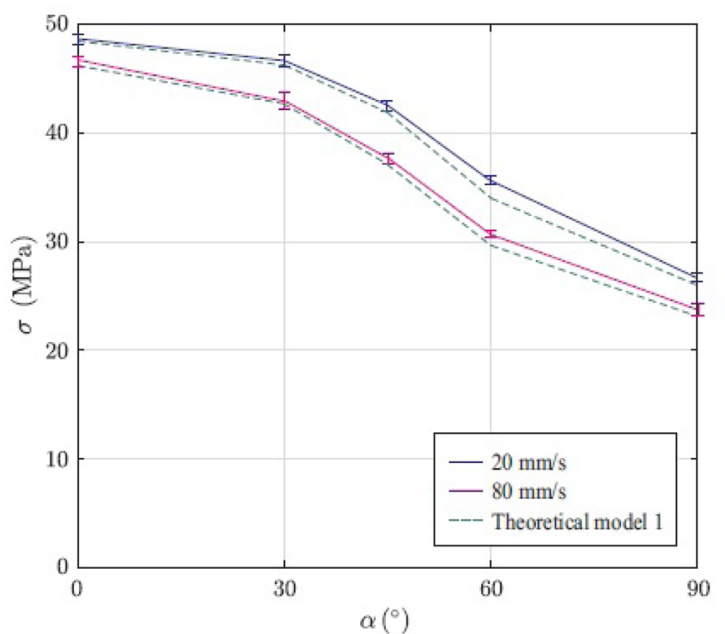

(a)

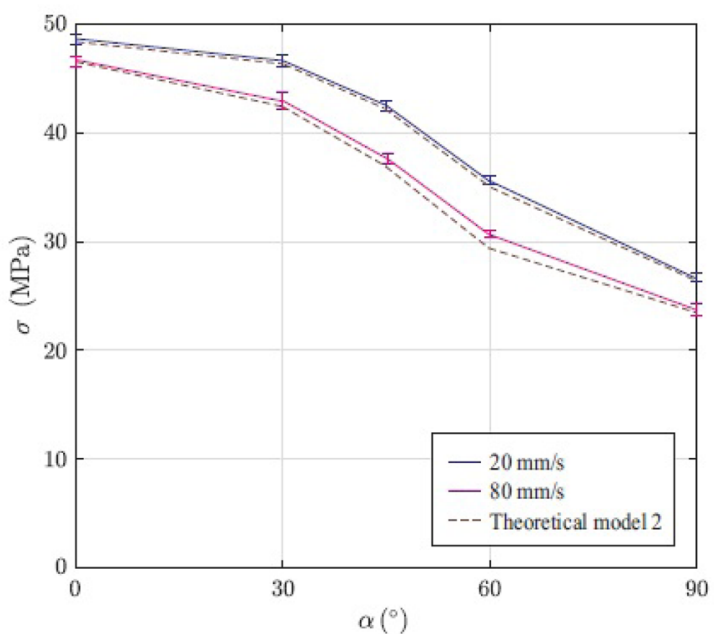

(b)

Figure 5. Comparison of the experimental results with (a) first and (b) second theoretical model.

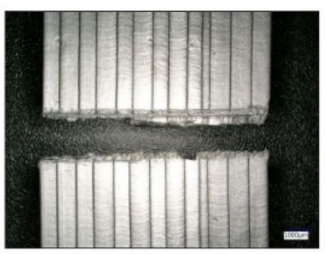

$\alpha=0^{\circ}$

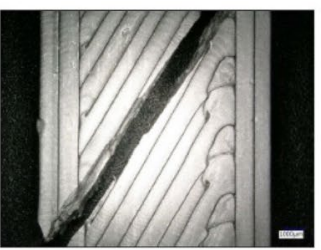

$\alpha=30^{\circ}$

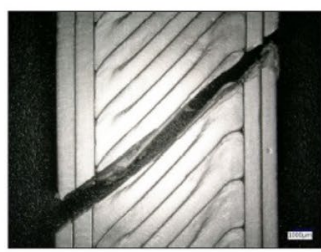

$\alpha=45^{\circ}$

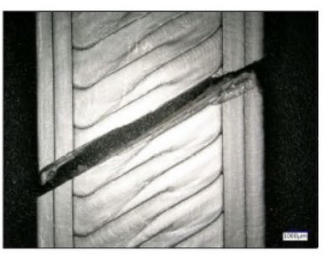

$\alpha=60^{\circ}$

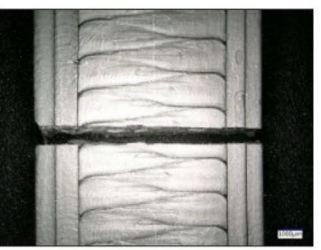

$\alpha=90^{\circ}$

Figure 6. Fractured 3D-printed specimens with different raster orientations.

the fracture. To this aim, a free-angle observation system was utilized for visual investigation of the fractured test coupons. Figure 6 shows fracture surfaces of examined specimens with different raster directions.

The visual inspection of fractured surfaces confirmed that in the $0^{\circ}$ specimens the crack is oriented vertically to the raster direction. In $30^{\circ}, 45^{\circ}$, and $60^{\circ}$ specimens, the fractures are oriented with raster directions.

\section{Theoretical background}

In the FDM process, 3D-printed parts are produced layer by layer, therefore, the parts can be considered as transverse isotropic materials. Indeed, each layer is isotropic materials in plane. Hence, the elastic constitutive relation of this material can be presented in the matrix form:

$$
\left[\begin{array}{l}
\varepsilon_{1} \\
\varepsilon_{2} \\
\varepsilon_{3} \\
\varepsilon_{4} \\
\varepsilon_{5} \\
\varepsilon_{6}
\end{array}\right]=\left[\begin{array}{cccccccc} 
& S_{11} & S_{12} & S_{12} & 0 & 0 & 0 & \\
& S_{12} & S_{22} & S_{23} & 0 & 0 & 0 & \\
& S_{12} & S_{23} & S_{22} & 0 & 0 & 0 & \\
0 & 0 & 0 & 2\left(S_{22}\right. & \left.-S_{23}\right) & 0 & 0 \\
0 & 0 & 0 & & 0 & & S_{55} & 0 \\
0 & 0 & 0 & & 0 & & 0 & S_{55}
\end{array}\right] \cdot\left[\begin{array}{c}
\sigma_{1} \\
\sigma_{2} \\
\sigma_{3} \\
\sigma_{4} \\
\sigma_{5} \\
\sigma_{6}
\end{array}\right] .
$$

As classically performed for the printed fiber ${ }^{44}$, we can assume the same mechanical behavior in two directions. In plane stress state $\left(\sigma_{3}=0, \tau_{23}=\sigma_{4}=0, \tau_{31}=\sigma_{5}=0\right)$, the constitutive equation can be presented as follows:

$$
\left[\begin{array}{c}
\varepsilon_{1} \\
\varepsilon_{2} \\
\gamma_{12}
\end{array}\right]=\left[\begin{array}{ccc}
S_{11} & S_{12} & 0 \\
S_{12} & S_{22} & 0 \\
0 & 0 & S_{55}
\end{array}\right]\left[\begin{array}{c}
\sigma_{1} \\
\sigma_{2} \\
\tau_{12}
\end{array}\right] .
$$

In Eq. (3), the flexibility coefficient matrix $S$ has four independent elastic constants: $S_{11}, S_{2}, S_{22}$ and $S_{55}$. These constants can be presented as functions of elastic modulus, Poisson's ratio, and shear modulus:

$$
S_{11}=\frac{1}{E_{1}}, S_{22}=\frac{1}{E_{2}}, S_{55}=\frac{1}{G_{12}}, S_{11}=\frac{-\vartheta_{21}}{E_{1}}=\frac{-\vartheta_{12}}{E_{2}} .
$$

Equation (2) can also be presented as follows to obtain stiffness matrix Q: 


$$
\left[\begin{array}{c}
\sigma_{1} \\
\sigma_{2} \\
\tau_{12}
\end{array}\right]=\left[\begin{array}{ccc}
Q_{11} & Q_{12} & 0 \\
Q_{12} & Q_{22} & 0 \\
0 & 0 & Q_{55}
\end{array}\right]\left[\begin{array}{c}
\varepsilon_{1} \\
\varepsilon_{2} \\
\gamma_{12}
\end{array}\right]
$$

The constitutive equation in different directions of materials can be presented as follows:

$$
\left[\begin{array}{c}
\sigma_{x} \\
\sigma_{y} \\
\tau_{x y}
\end{array}\right]=T^{-1}\left[\begin{array}{c}
\sigma_{1} \\
\sigma_{2} \\
\tau_{12}
\end{array}\right]=T^{-1} Q\left[\begin{array}{c}
\varepsilon_{1} \\
\varepsilon_{2} \\
\gamma_{12}
\end{array}\right]=T \quad Q\left(T^{-1}\right)^{T}\left[\begin{array}{c}
\varepsilon_{x} \\
\varepsilon_{y} \\
\gamma_{x y}
\end{array}\right],
$$

where $T$ is the stress rotation matrix which can be expressed as follows with respect to $\theta$ which is the angle between the global axis and the material axis:

$$
T=\left[\begin{array}{ccc}
\cos ^{2} \theta & \sin ^{2} \theta & 2 \sin \theta \cos \theta \\
\sin ^{2} \theta & \cos ^{2} \theta & -2 \sin \theta \cos \theta \\
-\sin \theta \cos \theta & \sin \theta \cos \theta & \cos ^{2} \theta-\sin ^{2} \theta
\end{array}\right]
$$

Hill-Tsai anisotropic yield criterion can be written as:

$$
\frac{\sigma_{1}^{2}}{X^{2}}+\frac{\sigma_{2}^{2}}{Y^{2}}-\frac{\sigma_{1} \sigma_{2}}{X^{2}}+\frac{\tau_{12}^{2}}{W^{2}}=1
$$

where $X, Y$, and $W$ are the ultimate tensile strength (UTS) in the principal axes of anisotropy. If there is only $\sigma_{x}$ in the loading direction of the test coupon, the stresses in the material reference system are as follows ${ }^{45}$ :

$$
\sigma_{1}=\sigma_{x} \cos ^{2} \theta, \sigma_{2}=\sigma_{x} \sin ^{2} \theta, \tau_{12}=\sigma_{x} \sin \theta \cos \theta .
$$

Combination of Eqs. (8) and (9) leads to:

$$
\sigma_{x}=T_{\theta}=\left[\frac{\cos ^{4} \theta}{X^{2}}+\left(\frac{1}{W^{2}}-\frac{1}{X^{2}}\right) \sin ^{2} \theta \cos ^{2} \theta+\frac{\sin ^{4} \theta}{Y^{2}}\right]^{-1 / 2},
$$

where $X$ and $Y$ denote the UTS of material in directions 1 and 2, respectively. Moreover, $W$ is ultimate shear strength of plane 12, and $T_{\theta}$ indicates the UTS of the load direction $\left(T_{0^{\circ}}=X\right.$ and $T_{90^{\circ}}=Y$ ) From Eq. (9), in plane shear strength can be determined by:

$$
W_{H T}=\left[\frac{1}{T_{\theta}^{2} \sin ^{2} \theta \cos ^{2} \theta}-\frac{1}{X^{2}} \cdot \frac{\cos ^{2} \theta}{\sin ^{2} \theta}-\frac{1}{Y^{2}} \cdot \frac{\sin ^{2} \theta}{\cos ^{2} \theta}+\frac{1}{X^{2}}\right]^{-1 / 2} .
$$

Based on the two following theoretical models, the UTS of FDM 3D-printed parts can be determined with respect to the printing direction:

- First model: considering Eq. (10) and shear strength of composite $\left(W_{s f}\right)$ the first model is as follows:

$$
T_{\theta}=\left[\frac{\cos ^{4} \theta}{X^{2}}+\left(\frac{1}{W_{s f}}-\frac{1}{X^{2}}\right) \sin ^{2} \theta \cos ^{2} \theta+\frac{\sin ^{4} \theta}{Y^{2}}\right]^{-1 / 2},
$$

where $W_{s f}$ is shear strength of composite; $W_{s f}=P_{45^{\circ}} / 2 b t$ and $P_{45^{\circ}}$ denotes ultimate bearing capacity of the test coupon with $45^{\circ}$ printing direction. Moreover, $\mathrm{b}$ and $\mathrm{t}$ indicate width and thickness of the specimen, respectively.

- Second model: Based on the Eqs. (10) and E(11), the UTS of loading direction can be determined as follows:

$$
T_{\theta}=\left[\frac{\cos ^{4} \theta}{X^{2}}+\left(\frac{1}{W_{H T}}-\frac{1}{X^{2}}\right) \sin ^{2} \theta \cos ^{2} \theta+\frac{\sin ^{4} \theta}{Y^{2}}\right]^{-1 / 2} .
$$

The last two equations, along with the UTS (X and Y) can be utilized to calculate the strength with respect to the printing angle.

\section{Experimental procedure}

Specimen preparation. In this study, PLA material was used for fabrication of the test coupons based on the FDM process. The specimens are designed according to ASTM D638 ${ }^{46}$. Considering the aim of this research, two printing parameters were changed during printing of the specimens: (a) raster orientation, and (b) printing speed. These parameters are defined as follows:

- Raster orientation: the angle $(\alpha)$ indicates the printing direction relative to the loading direction.

- Printing speed: the distance traveled by the extruder along the bed per unit time while extruding. After design of the dog-bone shaped specimens, 3D models were transferred into a slicer software and the output 


\begin{tabular}{|l|l|}
\hline Parameters & Values \\
\hline Nozzle temperature $\left({ }^{\circ} \mathrm{C}\right)$ & 215 \\
\hline Bed temperature $\left({ }^{\circ} \mathrm{C}\right)$ & 55 \\
\hline Printing speed $(\mathrm{mm} / \mathrm{s})$ & 20,80 \\
\hline Infill percentage $(\%)$ & 100 \\
\hline Density $\left(\mathrm{g} / \mathrm{cm}^{3}\right)$ & 1.24 \\
\hline Layer thickness $(\mathrm{mm})$ & 0.4 \\
\hline Number of contours & 2 \\
\hline Nozzle diameter $(\mathrm{mm})$ & 0.8 \\
\hline Extrusion width $(\mathrm{mm})$ & 1 \\
\hline Raster angle $\left({ }^{\circ}\right)$ & $0^{\circ}, 30^{\circ}, 45^{\circ}, 60^{\circ}, 90^{\circ}$ \\
\hline
\end{tabular}

Table 2. Printing parameters and properties of fabricated specimens.

\begin{tabular}{|c|c|c|c|c|}
\hline Material & Raster direction $\left({ }^{\circ}\right)$ & Printing speed $(\mathrm{mm} / \mathrm{s})$ & Failure load (N) & Displacement (mm) \\
\hline \multirow{10}{*}{ PLA } & \multirow{2}{*}{0} & 20 & 5285.9 & 6.9 \\
\hline & & 80 & 5149.3 & 6.1 \\
\hline & \multirow{2}{*}{30} & 20 & 4749.1 & 6.3 \\
\hline & & 80 & 4523.6 & 5.7 \\
\hline & \multirow{2}{*}{45} & 20 & 4138.2 & 5.8 \\
\hline & & 80 & 3849.3 & 5.1 \\
\hline & \multirow{2}{*}{60} & 20 & 3451.7 & 5.2 \\
\hline & & 80 & 3226.4 & 4.9 \\
\hline & \multirow{2}{*}{90} & 20 & 2793.5 & 5.1 \\
\hline & & 80 & 2638.2 & 4.8 \\
\hline
\end{tabular}

Table 3. The experimental failure loads and displacements of the examined 3D-printed specimens.

was saved as '.stl' format. Later, the models were printed utilizing FDM 3D printer. The printing parameters are presented in Table 2.

The specimens with unidirectional layup were printed in 8 layers with the total length of $160 \mathrm{~mm}$ and final thickness of $3.2 \mathrm{~mm}$. The specimens were designed and fabricated with two contours (outer layer that is surrounding the internal structure).

In the present study, the dog-bone shaped specimens were printed under two different printing speeds: $20 \mathrm{~mm} / \mathrm{s}$ and $80 \mathrm{~mm} / \mathrm{s}$. For each raster orientation and printing speed, six test coupons were fabricated. Later, the specimens were subjected to the tensile loads which is described in the following subsection.

Mechanical tests. A series of tensile tests was performed by using a hydraulic mechanical testing machine under temperature and relative humidity of $23 \pm 3{ }^{\circ} \mathrm{C}$ and $50 \pm 5 \%$, respectively. The machine The dog-bone shaped specimens with gauge length of $150 \mathrm{~mm}$ experienced quasi-static uniaxial tensile loads and their forcedisplacement curves were recorded at a constant crosshead speed of $5 \mathrm{~mm} / \mathrm{min}$. In the experimental practices, each examined specimen was fixed on the machine by an appropriate tensile fixture. It is noteworthy that, failure of the specimens was occurred at the gauge length of the test coupons. It means the documented breaking strength is accurately represent the actual breaking strength of the examined specimens. The 3D-printed specimens with different raster orientations underwent a series of tensile tests and the average of failure load and relevant displacements are presented in Table 3.

The obtained values presented in Table 3 showed that the failure load decreases while the raster angle is increased. Moreover, there is a gradual decrease in breaking force while printing speed is increased.

\section{Conclusion}

Experimental results demonstrated that raster direction has a significant influence on the strength of the specimens. In fact, the highest elastic modulus belongs to $0^{\circ}$ specimens. This value was gradually decreased with increase in the raster direction. Moreover, lowest ultimate tensile strength was reduced $53.76 \%$ in the specimen with the raster direction of $90^{\circ}$ printed at $80 \mathrm{~mm} / \mathrm{s}$. In this study, we have documented that strength was decreased according to increase of printing speed. In detail, higher speed can reduce the extrusion volume which decreased printing stability. Although printing speed has minor effect on strength of specimens (compared to 
raster direction), it has a crucial role on cost of production. However, combination of raster direction and printing speed showed a crucial effect on the strength and mechanical behavior of 3D-printed test coupons. Based on the conducted fractographic analysis we have documented that fractures are oriented with raster direction. Since in this study a biopolymer PLA was investigated, the obtained results can be used for analysis on biocomposite which used PLA with same grade. The obtained data can be used for new designs, reinforcement configurations, and next computational models.

\section{Data availability}

The datasets generated during and/or analysed during the current study are available from the corresponding author on reasonable request.

Received: 21 May 2021; Accepted: 3 January 2022

Published online: 19 January 2022

\section{References}

1. Kong, L., Ambrosi, A., Nasir, M. Z. M., Guan, J. \& Pumera, M. Selfpropelled 3D-printed aircraft carrier of light-powered smart micromachines for large-volume nitroaromatic explosives removal. Adv. Funct. Mater. 29, 1-9 (2019).

2. Bao, X. et al. 3D biomimetic artificial bone scaffolds with dual-cytokines spatiotemporal delivery for large weight-bearing bone defect repair. Sci. Rep. 7, 1-13 (2017).

3. Khosravani, M. R. \& Reinicke, T. 3D-printed sensors: Current progress and future challenges. Sens. Actuators A 305, 111916 (2020).

4. Zega, V. et al. The first 3-D-printed z-axis accelerometers with differential capacitive sensing. IEEE Sens. J. 18, 53-60 (2018).

5. Ma, L. et al. 3D printed personalized titanium plates improve clinical outcome in microwave ablation of bone tumors around the knee. Sci. Rep. 7, 1-10 (2017).

6. Nasiri, S. \& Khosravani, M. R. Progress and challenges in fabrication of wearable sensors for health monitoring. Sens. Actuators A 312, 112105 (2020).

7. Yang, C. et al. Influence of thermal processing conditions in $3 \mathrm{D}$ printing on the crystallinity and mechanical properties of PEEK material. J. Mater. Process. Technol. 248, 1-7 (2017).

8. Mohamed, O. A., Masood, S. H. \& Bhowmik, J. L. Experimental investigation of time dependent mechanical properties of PC-ABS prototypes processed by FDM additive manufacturing process. Mater. Lett. 193, 58-62 (2017).

9. Jiang, J., Lou, J. \& Hu, G. Effect of support on printed properties in fused deposition modelling processes. Virtual Phys. Prototyp. 14, 308-315 (2019).

10. Sharma, A. \& Ahn, B. Vacum brazing of $\mathrm{Al}_{2} \mathrm{O}_{3}$ and 3D printed Ti6Al4V lap-joint using high entropy driven AlZnCuFeSi filler. Sci. Rep. 11, 1-15 (2021).

11. Porter, J. H., Cain, T. M., Fox, S. L. \& Harvey, P. S. Influence of infill properties on flexural rigidity of 3D-printed structural members. Virtual Phys. Prototyp. 14, 148-159 (2019).

12. Berzal, M., Barajas, C., del Mazo, D., Caja, C. \& Maresca, P. Simple filling patterns to model mechanical behaviour of FDM’s test pieces under torsion. Proc. Manuf. 29, 1-9 (2017).

13. Ren, L. et al. 3D printing of materials with spatially non-linearly varying properties. Mater. Des. 156, 470-479 (2018).

14. Costas, M., Morin, D., Lucio, M. D. \& Langseth, M. Testing and simulation of additively manufactured AlSi10Mg components under quasi-static loading. Eur. J. Mech. A. Solids 81, 103966 (2020).

15. Nachtane, M. et al. Experimental investigation on the dynamic behavior of 3D printed CF-PEKK composite under cyclic uniaxial compression. Compos. Struct. 247, 112474 (2020).

16. Ahmed, A. A. \& Susmel, L. Additively manufactured PLA under static loading: Strength/cracking behaviour vs. deposition angle. Proc. Struct. Integr. 3, 498-507 (2017).

17. Aliheidari, N., Christ, J. F., Tripuraneni, R., Nadimpalli, S. \& Ameli, A. Interlayer adhesion and fracture resistance of polymers printed through melt extrusion additive manufacturing process. Mater. Des. 156, 351-361 (2018).

18. Khosravani, M. R. \& Zolfagharian, A. Fracture and load-carrying capacity of 3D-printed cracked components. Extreme Mech. Lett. 37, 100692 (2020).

19. Kiendl, J. \& Gao, C. Controlling toughness and strength of FDM 3Dprinted PLA components through the raster layup. Compos. Part B 180, 107562 (2020).

20. Ezeh, O. H. \& Susmel, L. On the notch fatigue strength of additively manufactured polylactide (PLA). Int. J. Fatigue 136, 105583 (2020).

21. Khosravani, M. R., Berto, F., Ayatollahi, M. R. \& Reinicke, T. Fracture behavior of additively manufactured components: A review. Theor. Appl. Fract. Mech. 109, 102763 (2020).

22. Croccolo, D., De Agostinis, M. \& Olmi, G. Experimental characterization and analytical modelling of the mechanical behaviour of fused deposition processed parts made of ABS-M30. Comput. Mater. Sci 79, 506-518 (2013).

23. Fatemi, A., Molaei, R., Sharifimehr, S., Phan, N. \& Shamsaei, N. Multiaxial fatigue behavior of wrought and additive manufactured Ti-6Al-4V including surface finish effect. Int. J. Fatigue 100, 347-366 (2017).

24. Ng, C. T. \& Susmel, L. Notch static strength of additively manufactured acrylonitrile butadiene styrene (ABS). Addit. Manuf. 34, $101212(2020)$.

25. Linul, E., Marsavina, L. \& Stoia, D. I. Mode I and II fracture toughness investigation of laser-sintered polyamide. Theor. Appl. Fract. Mech. 106, 102497 (2020).

26. Khosravani, M. R., Zolfagharian, A., Jennings, M. \& Reinicke, T. Structural performance of 3D-printed composites under various loads and environmental conditions. Polym. Test 91,106770 (2020).

27. Allum, J., Gleadall, A. \& Silberschmidt, V. V. Fracture of 3D-printed polymers: Crucial role of filament-scale geometric features. Eng. Fract. Mech. 224, 1068181 (2020).

28. Jiang, J. et al. Analysis and prediction of printable bridge length in fused deposition modelling based on back propagation neural network. Virtual Phys. Prototyp. 14, 253-266 (2019).

29. Nasiri, S. \& Khosravani, M. R. Machine learning in predicting mechanical behavior of additively manufactured parts. J. Mater. Res. Technol. 14, 1137-1153 (2021).

30. Lin, W. et al. Single-layer temperature-adjusting transition method to improve the bond strength of 3D-printed PCL/PLA parts. Compos. A 115, 22-30 (2018).

31. Bhandari, S., Lopez-Anido, R. A. \& Gardner, D. J. Enhancing the interlayer tensile strength of 3D printed short carbon fiber reinforced PETG and PLA composites via annealing. Addit. Manuf. 30, 100922 (2019).

32. Colombo, C. et al. Modulating the damping capacity of SLMed AlSi10Mg trough stress-relieving thermal treatments. Theor. Appl. Fract. Mech. 107, 102537 (2020).

33. Sang, W. L., Jian, X. \& Wang, J. Influence of sanding and plasma treatment on shear bond strength of 3D-printed PEI, PEEK and PEEK/CF. Int. J. Adhes. Adhes. 100, 102614 (2020). 
34. Chacon, J. M., Caminero, M. A., Garcia-Plaza, E. \& Nunez, P. J. Additive manufacturing of PLA structures using fused deposition modelling: Effect of process parameters on mechanical properties and their optimal selection. Mater. Des. 124, 143-157 (2017).

35. Ning, F., Cong, W., Hu, Y. \& Wang, H. Additive manufacturing of Carbon fiber-reinforced plastic composites using fused deposition modeling: Effects of process parameterson tensile properties. J. Compos. Mater. 51, 451-462 (2017).

36. Shariatnia, S., Veldana, A., Obeidat, S., Jarrahbashi, D. \& Asadi, A. Atomization of cellulose nanocrystals aqueous suspensions in fused deposition modeling: A scalable technique to improve the strength of 3D printed polymers. Compos. Part B 177, 107291 (2019).

37. Ayatollahi, M. R., Nabavi-Kivi, A., Bahrami, B., Yahya, M. Y. \& Khosravani, M. R. The influence of in-plane raster angle on tensile and fracture strengths of 3D-printed PLA specimens. Eng. Fract. Mech. 237, 107225 (2020).

38. Custodio, C. L. et al. Powder loading effects on the physcicochemical and mechanical properties of 3D printed poly lactic acid/ hydroxyapatite biocomposites. Int. J. Bioprinting. 7, 112-122 (2021).

39. Wang, P., Zou, B., Ding, S., Li, L. \& Huang, C. Effects of FDM-3D printing parameters on mechanical properties and microstructure of CF/PEEK and GF/PEEK. Chin. J. Aeronaut. 33, 236-246 (2020).

40. Murugan, V., Marconi, S., Alaimo, G. \& Auricchio, F. A non-standard slicing pattern for 3D-printing of structural components. In International Conference on Adaptive Modeling and Simulation. Spain (2019).

41. Gebisa, A. W. \& Lemu, H. G. Influence of 3D printing FDM process parameters on tensile property of ULTEM 9085. Proc. Manuf. 30, 331-338 (2019).

42. Cattenone, A., Morganti, S., Alaimo, G. \& Auricchio, F. Finite element analysis of additive manufacturing based on fused deposition modeling: Distortions prediction and comparison with experimental data. ASME J. Manuf. Sci. Eng. 141, 011010 (2019).

43. Bermudez, D. et al. A Comparison of the physical properties of two commercial 3D printing PLA grades. Virtual Phys. Prototyp. 16, 178-195 (2021).

44. Rodriguez, J. F., Thomas, J. P. \& Renaud, J. E. Design of fused-deposition ABS components for stiffness and strength. J. Mech. Des. 125, 545-551 (2003).

45. Alaimo, G., Marconi, S., Costato, L. \& Auricchio, F. Influence of mesostructured and chemical composition on FDM 3D-printed parts. Compos. B 113, 371-380 (2017).

46. ASTM D638-14 Standard Test Method for Tensile Properties of Plastics, Standard, American Society for Testing Materials, West Conshohocken, USA (2014).

\section{Acknowledgements}

This work as part of the project "Smart Production Design Center" (SmaP) is funded by the European Regional Development Fund (ERDF) under the program OP EFRE NRW 20142020 (EFRE-0200545).

\section{Author contributions}

M.R.K.: Conceptualization, Methodology, Writing_Original Draft, Visualization. F.B.: Writing—Review \& Editing. M.R.A.: Writing-Review \& Editing. T.R.: Funding acquisition, Writing-Review \& Editing. All authors reviewed the manuscript.

\section{Funding}

Open Access funding enabled and organized by Projekt DEAL.

\section{Competing interests}

The authors declare no competing interests.

\section{Additional information}

Correspondence and requests for materials should be addressed to M.R.K.

Reprints and permissions information is available at www.nature.com/reprints.

Publisher's note Springer Nature remains neutral with regard to jurisdictional claims in published maps and institutional affiliations.

(c) (i) Open Access This article is licensed under a Creative Commons Attribution 4.0 International License, which permits use, sharing, adaptation, distribution and reproduction in any medium or format, as long as you give appropriate credit to the original author(s) and the source, provide a link to the Creative Commons licence, and indicate if changes were made. The images or other third party material in this article are included in the article's Creative Commons licence, unless indicated otherwise in a credit line to the material. If material is not included in the article's Creative Commons licence and your intended use is not permitted by statutory regulation or exceeds the permitted use, you will need to obtain permission directly from the copyright holder. To view a copy of this licence, visit http://creativecommons.org/licenses/by/4.0/.

(c) The Author(s) 2022 